\title{
Harnessing Immune Switch Responses in Relapse of Ovarian Cancer: All Signals Needed
}

\section{Cheng-Tao Lin ${ }^{1 *}, 2$}

${ }^{1}$ Department of Obstetrics and Gynecology, Chang Gung Memorial Hospital, Chang Gung University, Taoyuan, Taiwan

${ }^{2}$ Chang Gung Immunology Consortium, Chang Gung Memorial Hospital, Chang Gung University, Gueishan, Taoyuan, Taiwan

\section{Introduction}

The immune system plays an important role in controlling and elimination of cancer. Persistent infection and/or inflammation leads to gradual accumulation of tolerant apoptoic or immunosenescence cells within host body. Timely, how to trigger antigen-presenting cells generate and/or amplify host immunosurveillance after optimal debulking operation to be a central role. Advanced invasive ovarian cancer after conventional therapy, they still faced $60-70 \%$ disease relapse. Once relapse, they suffered from detrimental outcome following first-line treatment. Thus, clinician has to do early imaging and/or tumor markers' screening as well as possible and early intervention of integrated therapy for relapse of ovarian cancer.

Clinician has to educate relapse of cancer patient and/or families to tolerate and understand common cold-like fever to elicit "signal 0 , to mimic anger signal" following immunomodulatory therapy [1]. OK432 one vial administration of subcutaneous skin to trigger dendritic cells' (DC) activation and present innate Toll-like receptor to capture cancer-associated antigen to solicit Toll-like receptor (TLR) 4-MD2 signal pathway. Ok-432 as adjuvant agent trigger DCs reside in tissue or peritoneal macrophages to capture antigen to form an efficient vaccine to link innate cells and adaptive immune cells to elicit host immunosurveillance [2].

Ok-432 is a useful adjuvant in DC-based anticancer immunotherapy. Administration of subcutaneous OK-432 injection to activate antigen presenting cells including macrophages and/or dendtritic cells to secret multiple cytokines to generate efficient anti-cancer response. The effects of OK-432 on DCs recognize cancer-associated antigens (vaccines) to mimic signal 1 and secrete cytokines (e.g. IL-12, IL-18, IFN-alpha and interferon-gamma production) directly through Toll-like receptor, and adaptive cells' receptor and stimulation of costimulatory molecules (e.g. CD40, CD80, CD83, CD86, HLA-DR, etc.) to mimic signal 2 to generate a vaccine [2]. IL-2 is a cytokine for T lymphocytes' growth factor to mimic signal 3. IL-2 treatment triggers chemokine receptor CXCR4 expression on Treg cells, enables Treg cells migration toward chemokine CXCL12 in tumor microenvironment, and may enforce Treg cell tumor accumulation [3]. Type I interferons (alpha/beta) keep activated $\mathrm{T}$ cells alive and create a bridge between innate immune cell and adaptive immune cells cell-on-cell interaction. IFNs may exhibit important antitumoral and antiangiogenetic effects and induces apoptosis in malignant cells [4].

A single administration of cyclophosphamide was shown to deplete CD4+CD25+ T cells in tumor-bearing animals, delay tumor growth, and cure rats bearing established tumors when followed by an immunotherapy which has no curative effect when administered alone. Both host immunologic and intraperitoneal micrometastastic factors have been examined with immunomodulatory therapy (IMT) or bevacizumab (avastin). Subcutaneous administered IMT switch type 2 immune cells to type 1 immunosurveillance cells. Intraperitoneal avastin administered to neutralize aberrant VEGF expression to enhance conventional chemotherapy and/radiotherapy for relapse of ovarian cancer [4].

\section{Case Demonstration}

A case birth on August 1968 female, Gravida 2 Paravida 2, suffered from general abdominal painful and tenderness in 2009. A 44-yearold female diagnosed with advanced ovarian cancer stage 4 with liver metastasis was treated with suboptimal debulking surgery and received conventional chemotherapy. Within one year, on May 2010, she had abnormal CA-125 increased and PET-CT showed peritoneal carcinomatosis. She again received hyperthermia and intraperitoneal chemotherapy during operation. Due to CA-125 still high level, she had bowel obstruction on December 2011. She requested to do immunomodulatory therapy to want to achieve "proof-of concept" to control her peritoneal carcinomatosis scerions on January 2012. The priming immunomodulatory agents include picibanil (OK-432) at dayl and aldeleukin (A: interleukin-2) at day 2. She received celecoxib twice tablet daily. 2 days later, she agreed to do more alternatively immunomodulatory therapy as booster immunization. No fever onset, 24 to 48 hours later, I administered low dose chemotherapy one vial such as paclitaxol or lipodoxil to create host immunosurveillance and hold cancer cell progression or avoid host immunocompromised status. She had progression free interval for over 12 months and/or better life span with normalization tumor marker to make her better spirit after immunomodulatory therapy (Figures 1 and 2).

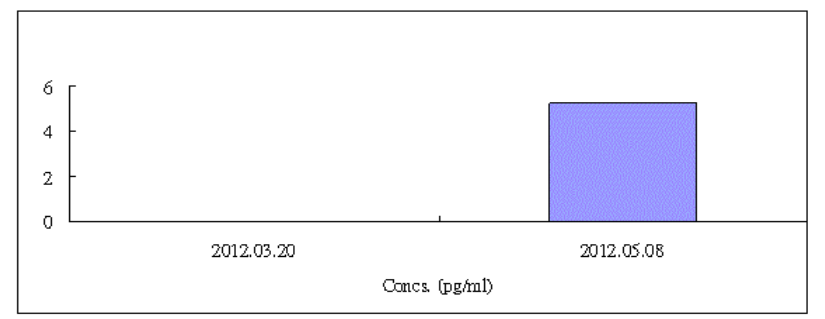

Figure 1: She received immunomodulatory therapy (OA priming and booster) to trigger host immunosurveillance to secrete interleukin-12 to elevate over 4 fold through ELISA method compared to threshold of conventional therapy.

*Corresponding author: Cheng-Tao Lin, Department of Obstetrics and Gynecology, Chang Gung Memorial Hospital and Chang Gung University, Taoyuan, Taiwan, Tel: +886-3-3184301; E-mail: 51424@cgmh.org.tw

Received January 11, 2016; Accepted April 20, 2016; Published April 27, 2016

Citation: Lin CT (2016) Harnessing Immune Switch Responses in Relapse of Ovarian Cancer: All Signals Needed. J Nucl Med Radiat Ther 7: 286. doi:10.4172/2155-9619.1000286

Copyright: (c) 2016 Lin CT. This is an open-access article distributed under the terms of the Creative Commons Attribution License, which permits unrestricted use, distribution, and reproduction in any medium, provided the original author and source are credited. 
Citation: Lin CT (2016) Harnessing Immune Switch Responses in Relapse of Ovarian Cancer: All Signals Needed. J Nucl Med Radiat Ther 7: 286. doi:10.4172/2155-9619.1000286

Page 2 of 2

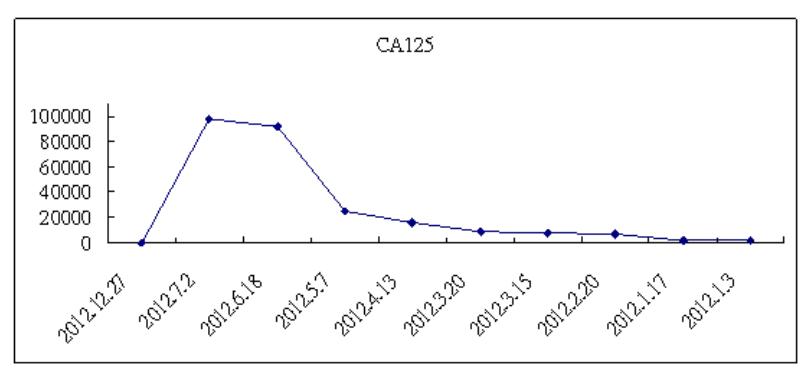

Figure 2: We hold and persistent suppress patient's to worry about stabilization of tumor marker CA-125.

\section{Acknowledgments}

The study was supported by grants CRRPG3D0051-3 and CMRPG3F0251, which were provided by Chang Gung University Hospital (Taoyuan, Taiwan).

\section{References}

1. Chen CY, Lai CH, Yang LY, Tang YH, Chou HH, et al. (2015) Immunomodulatory therapy in refractory/recurrent ovarian cancer. Taiwan J Obstet Gynecol 54: 143-149.

2. Chen IJ, Yen CF, Lin KJ, Lee CL, Soong YK, et al. (2011) Vaccination with OK432 followed by TC-1 tumor lysate leads to significant antitumor effects. Reprod Sci 18: 687-694.

3. Lin CT, Tsai YC, He L, Yeh CN, Chang TC, et al. (2007) DNA vaccines encoding IL-2 linked to HPV-16 E7 antigen generate enhanced E7-specific CTL responses and antitumor activity. Immunol Lett 114: 86-93.

4. Lin CT, Wang CN, Lai CH (2013) Immunotherapy for advanced or relapsed cervical cancer Gynecology and Minimally invasive therapy. GMIT 2: 3-7 